\section{Early division of a modified Cutler- Beard flap with a free tarsal graft}

\begin{abstract}
Aims We describe a variation of the CutlerBeard flap in the reconstruction of upper eyelid defects.

Methods The technique of upper eyelid reconstruction with a free tarsal graft and a cutaneous lower eyelid advancement flap divided at 2 weeks is described. Four cases where this technique was used for reconstruction of eyelid defects due to periocular malignancy are also reported. Results There were three male and one female patients ranging in age from 61 to 78 years. The underlying diagnoses were squamous cell carcinoma, trichilemmal carcinoma, and two basal cell carcinomas. Follow-up of 6-28 months revealed a good outcome in all four cases with one patient developing a mild cicatricial lower eyelid ectropion that was managed conservatively.

Conclusions A modified Cutler-Beard flap with free tarsal graft and early division may provide an effective alternative for upper eyelid reconstruction in cases with sufficient lower eyelid skin laxity.
\end{abstract}

Oculoplastic \& Orbital Unit Department of Ophthalmology Royal Adelaide Hospital University of Adelaide Australia

Correspondence: D Selva Oculoplastic \& Orbital Unit Department of

Ophthalmology

Royal Adelaide Hospital

North Terrace

Adelaide, SA 5000

Australia

Tel: + 61882225222

Fax +61882225221

E-mail: Awestwoo@

mail.rah.sa.gov.au

Received: 2 June 2003 Accepted in revised form: 24 July 2003

Published online: 6 February 2004

Proprietary interest: None
Eye (2004) 18, 714-717. doi:10.1038/sj.eye.6701321

Published online 6 February 2004

Keywords: Cutler-Beard; bridging flap; free tarsal graft; upper lid reconstruction

\section{Introduction}

The reconstruction of an upper lid defect is often challenging, and there are a number of considerations that will affect the choice of procedure. The upper lid's principle role in protecting the ocular surface requires apposition of a mucosal surface, ideally conjunctiva, to the cornea. To refresh the tear clear of the visual axis, without difficulty. The film, the lid needs to be able to close, and open
J Hsuan and D Selva

margin should be stable to prevent stratified squamous epithelium, lashes, or hairs from irritating the cornea. In addition to these functional aspects, the cosmetic appearance of the upper lid is highly important, with potentially significant psychological or social morbidity arising from a poorly reconstructed lid. In general, the tissues used should match the original as closely as possible, and disruption to normal structures should be minimised. Two-stage bridging flaps requiring prolonged closure of the eye can be disabling for the patient, especially if the sight of the fellow eye is poor.

Small defects of the upper lid of up to a quarter of its width, or a third if there is sufficient tissue laxity, can be closed directly. Larger defects require the use of some form of flap and/or a free graft. A Cutler-Beard flap is commonly used for large upper eyelid defects and is a cutaneoconjunctival lower eyelid advancement flap, traditionally divided at around 8 weeks. ${ }^{1}$ We describe a variation involving a free tarsal graft with a skin-only advancement flap from the lower eyelid, which is divided at 2 weeks.

\section{Methods}

Four cases of upper eyelid malignancy requiring full-thickness eyelid reconstruction are reported. A modified Cutler-Beard flap with a free tarsal graft was performed on all patients by the senior author (DS). Informed consent was obtained from all patients after the procedure had been fully explained.

\section{Surgical technique}

A free tarsal graft is harvested from the contralateral upper eyelid and sutured into the posterior lamella of the upper eyelid defect using 6/0 vicryl. The edge of the levator 
aponeurosis is identified and sutured to the superior margin of the tarsal graft. A full-thickness horizontal incision corresponding to the width of the graft is made below the tarsus of the lower eyelid. A skin flap is raised and passed through the conjunctival incision under the lower eyelid margin to be sutured to the skin edges of the upper eyelid defect (Figure 1). A firm pad is applied for 2 days. The skin flap is divided at 2 weeks and reset into the lower eyelid. If necessary, the skin and tarsal edges of the new upper lid margin are sculpted to achieve a satisfactory contour, but no further sutures are required.

\section{Results}

The details of the four cases are outlined in Table 1. All patients had biopsy proven malignancies of the upper eyelid that were excised by Mohs' micrographic surgery. This resulted in full-thickness defects that involved the

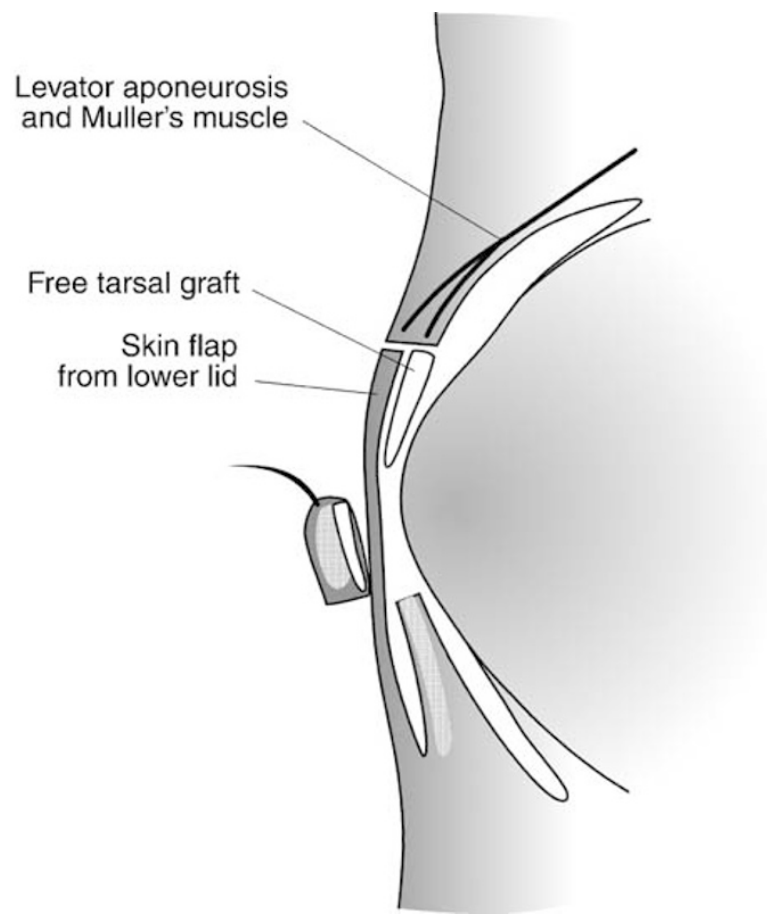

Figure 1 Diagram to illustrate the skin bridging flap majority of the eyelid. In all cases, the remaining upper eyelid skin was insufficient for advancement over a free tarsal graft and it was thought that the lower eyelid skin laxity would be adequate to enable an advancement flap. Reconstruction in each case was by the technique described above (Figure 2). In one patient, case 3, the defect also involved the lateral canthus and the lateral $4 \mathrm{~mm}$ of the lower eyelid. In this case, the free tarsal graft in the upper eyelid was sutured to a periosteal flap from the lateral orbital rim, and the remaining lower eyelid was sutured to the periosteum of the lateral rim prior to the lower eyelid advancement flap being constructed. In each case, there was no donor site morbidity in the contralateral upper eyelids and no necrosis or tissue loss following division of the flap. At the time of division, there was brisk bleeding from the upper eyelid margin suggestive of good vascularity. In no case was there entropion of the reconstructed upper eyelid. One patient, case 1, developed a mild cicatricial ectropion of the lower eyelid following resetting of the skin flap (Figure $2 \mathrm{~d}$ ).

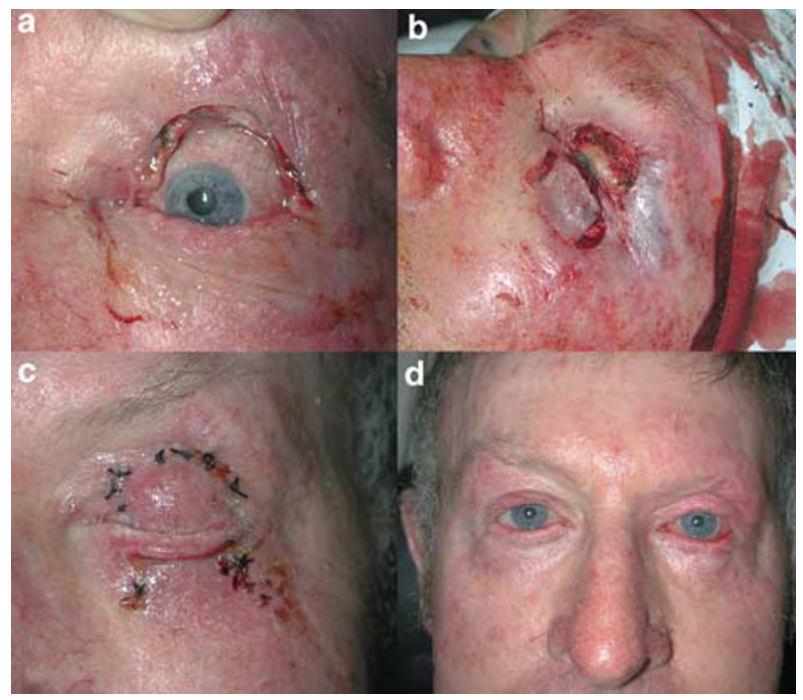

Figure 2 Case 1. (a) Large full-thickness upper lid defect following Mohs' excision of a trichilemmal carcinoma; (b) posterior lamella reconstructed with free tarsal graft, skin flap fashioned from lower lid; (c) skin flap sutured in place; (d) mild lower lid ectropion following division of flap at 2 weeks

Table 1 Patient data

\begin{tabular}{|c|c|c|c|c|c|c|}
\hline Case & Age(years)/Gender & Laterality & Diagnosis $^{a}$ & Defect size $(\mathrm{mm})$ & $\begin{array}{c}\text { Follow-up } \\
\text { (months) }\end{array}$ & Complications \\
\hline 1 & $61 / \mathrm{M}$ & Left & TLC & $20 \times 12$ & 14 & $\begin{array}{l}\text { Mild LL } \\
\text { ectropion }\end{array}$ \\
\hline 2 & $65 / \mathrm{M}$ & Right & SCC & $19 \times 9$ & 6 & \\
\hline 3 & $69 / \mathrm{F}$ & Left & $\mathrm{BCC}$ & $22 \times 10$ & 28 & \\
\hline 4 & $78 / \mathrm{M}$ & Left & $\mathrm{BCC}$ & $17 \times 9$ & 21 & \\
\hline
\end{tabular}

aTLC, trichilemmal carcinoma; SCC, squamous cell carcinoma; BCC, basal cell carcinoma. 
As he was asymptomatic, this was managed conservatively. Follow-up ranged from 6 to 28 months and all patients were comfortable with no upper eyelid retraction, lagophthalmos, or corneal exposure. The margin-reflex distances are shown in Table 2, with the postoperative lid position remaining within $1 \mathrm{~mm}$ of the preoperative position in all cases.

\section{Discussion}

In 1955, Cutler and Beard ${ }^{1}$ described the use of a fullthickness lower lid bridging flap to reconstruct large upper lid defects. They reported 12 cases with the advancement flap taken from below the lower tarsal border. The only significant complication they noted was lower lid retraction. However, with this procedure there is no rigid structural support for the posterior lamella, and other authors reported stability problems with the reconstructed upper lid, with posterior shrinkage leading to entropion and ocular irritiation. ${ }^{2}$ To overcome this problem, Mauriello and Antonacci ${ }^{3}$ advocated a reverse Hughes type procedure, taking a tarsoconjunctival flap from the lower lid to reform the posterior lamella of the upper lid, covered by a free skin graft. Leone ${ }^{4}$ earlier suggested a similar procedure, but combined it with inferior advancement of the residual superior tarsus, joining the two tarsoconjunctival flaps to reform the posterior lamella. This technique was later criticised by Jordan et $a l^{5}$, who advocated advancing the residual upper lid tarsus alone, thus negating the need for a bridging flap. Avoiding a two-stage procedure and closure of the eye are preferable when possible, but Jordan states this requires at least $3 \mathrm{~mm}$ of residual superior tarsus. With Mohs' micrographic surgery, there is often relative sparing of the posterior lamella, but there remain those who have insufficient or no residual tarsus that require some other source of rigid support. Wesley and $\mathrm{McCord}^{6}$ reported good results in 26 patients who received donor sclera, sandwiched between the orbicularis and conjunctiva in a Cutler-Beard bridging flap, with no patient developing entropion. Carrol $^{2}$ suggested that sclera may undergo significant shrinkage, proposing ear cartilage may be more resilient. Other

Table 2 Preoperative and postoperative margin reflex distances (mm)

\begin{tabular}{lllll}
\hline Case & $\begin{array}{l}\text { Pre-op } \\
u M R D\end{array}$ & $\begin{array}{l}\text { Pre-op } \\
l M R D\end{array}$ & $\begin{array}{l}\text { Post-op } \\
\text { uMRD }\end{array}$ & $\begin{array}{l}\text { Post-op } \\
l M R D\end{array}$ \\
\hline 1 & R3 L3 & R6 L6 & R3 L3 & R6 L5 \\
2 & R4 L4 & R5 L5 & R3 L4 & R5 L5 \\
3 & R3 L2 & R6 L6 & R3 L2 & R6 L5 \\
4 & R2 L2 & R6 L6 & R2 L3 & R6 L6 \\
\hline
\end{tabular}

tissues have also been proposed, but it is reasonable to argue that the best substitute for the posterior lamella is autogenous tarsal plate and conjunctiva. This may be safely harvested as a free tarsal graft from the opposite upper lid, or as a rotational flap as described by Kersten et al. ${ }^{7}$ It may be possible to advance a myocutaneous flap or skin flap from neighbouring tissue if there is sufficient excess, ${ }^{8}$ or else a bridging flap of orbicularis and skin can been used, a modified Cutler-Beard.

Our technique is a development of this method, but using skin alone in the advancement flap. This spares dissection of the orbicularis resulting in less disruption to the lower lid tissues. We suggest that the time required for the skin to stretch would be less than that for a comparable myocutaneous flap. This is supported by our results where the flap was divided at 2 weeks. The skin-only flap carries a sufficient blood supply, and the patient's eye is closed for only 2 weeks, compared to the traditional 4-8 weeks. Vascularisation of the tarsal graft appears to occur more quickly than was originally thought. A comparable situation occurs in the Hughes flap, which was traditionally left for 6 weeks. McNab et $a l^{9}$ showed that there was no difference in upper or lower lid position in patients divided at 2 or 4 weeks, and vascularisation was established by 2 weeks. Bartley and Messenger ${ }^{10}$ described good outcomes in eight patients with partial or complete traumatic dehiscence of Hughes flaps between 1 and 11 days after surgery. In our series, all patients had their flaps divided at 2 weeks, and showed bleeding from the reconstructed tarsal margin, demonstrating good vascularity.

The problem of lower lid ectropion is common to all Cutler-Beard type procedures. The main determinants are the skin tension of the lower eyelid and upper cheek, and the degree of horizontal laxity of the lower lid margin. Only one of our patients developed a mild ectropion despite dividing the flap at 2 weeks. Not surprisingly, this patient required the greatest vertical displacement to cover the upper lid defect, and was the youngest. In most patients, therefore, 2 weeks is adequate, but where there is a large defect, or the lower lid skin is tight, especially in younger patients, it may be necessary to wait longer for the skin to stretch. Other surgical options that may be utilised are a lateral tarsal strip if there is horizontal laxity of the lower lid, or a periosteal anchoring suture, as suggested by Harris and Perez, ${ }^{11}$ to reduce the downward tractional force of the mobilised flap.

\section{Conclusion}

The results of this small study show that a lower lid skin only bridging flap combined with a free tarsal graft is a useful alternative technique for the reconstruction of large upper lid defects. The method has the advantage of 
less disruption to the lower lid and early division of the flap, with good function and cosmesis of the reconstructed upper lid.

\section{References}

1 Cutler NL, Beard C. A method for partial and total upper lid reconstruction. Am J Ophthalmol 1955; 39: 1-7.

2 Carrol RP. Entropion following the Cutler-Beard procedure. Ophthalmology 1983; 90: 1052-1055.

3 Mauriello JA, Antonacci R. Single tarsoconjunctival flap (lower eyelid) for upper eyelid reconstruction ('reverse' modified Hughes procedure). Ophthalmic Surg 1994; 25: 374-378.

4 Leone CR. Tarsal-conjunctival advancement flaps for upper eyelid reconstruction. Arch Ophthalmol 1983; 101: 945-948.

5 Jordan DR, Anderson RL, Nowinski TS. Tarsoconjunctival flap for upper eyelid reconstruction. Arch Ophthalmol 1989; 107: 599-603.
6 Wesley RE, McCord CD. Transplantation of eyebank sclera in the Cutler-Beard method of upper eyelid reconstruction. Ophthalmology 1980; 87: 1022-1028.

7 Kersten RC, Anderson RL, Tse DT, Weinstein GL. Tarsal rotational flap for upper eyelid reconstruction. Arch Ophthalmol 1986; 104: 918-922.

8 Anderson RL, Edwards JJ. Reconstruction by myocutaneous eyelid flaps. Arch Ophthalmol 1979; 97: 2358-2362.

9 McNab AA, Martin P, Benger R, O'Donnell B, Kourt G. A prospective randomized study comparing division of the pedicle of modified Hughes flaps at two or four weeks. Ophthalmic Plast Reconstr Surg 2001; 17: 317-319.

10 Bartley GB, Messenger MM. The dehiscent Hughes flap: outcomes and implications. Trans Am Ophthalmol Soc 2002; 100: 61-66.

11 Harris GJ, Perez N. Anchored flaps in post-Mohs reconstruction of the lower eyelid, cheek, and lateral canthus: avoiding eyelid distortion. Ophthalmic Plast Reconstr Surg 2003; 19: 5-13. 